Ю.В. Стасєв, А.Ю. Сійчук, К.Ю. Костиленко, О.В. Манакова

Харківський Наџіональний університет Повітряних Сил ім. І. Кожедуба, Харків

\title{
АЛГОРИТМИ ПОБУДОВИ ДИСКРЕТНИХ ОРТОГОНАЛЬНИХ СИГНАЛІВ НА ОСНОВІ МАТРИЦЬ АДАМАРА
}

В статті запропоновані алгоритми побудови ортогональних систем сигналів на основі матриць Адамара з числом елементів $L \equiv 0($ mod4). Реалізація цүих алгоритмів дозволяє більи гнучко використовувати дискретні сигнали в випадках зміни енергетичних, часових та частотних ресурсів систем зв'язку та управління. В статті сформульовані та доведені твердження, щяо є математичною основою побудови ортогональних сигналів з числом елементів $L \equiv 0(\bmod 4)$. Показано, щзо практично для всіх значень $L \equiv 0(\bmod 4)$ icнує алгоритм побудови ортогональних сигналів. Алгоритми побудови матриць Адамара за різних значень L 6 числі випадків мають суттєві відмінності. Аналіз способів і алгоритмів побудови ОДС дозволяє зробити такі висновки, щуо для абсолютної більшості значень існують матриџі Адамара, аватми побудови матриць Адамара за різних значень L в числі випадків мають суттєві відмінності. Аналіз способів $і$ алгоритмів побудови ОДС дозволяє зробити такі висновки, щуо для абсолютної більшості значень існують матриці Адамара, алгоритми побудови матрищь Адамара для різних значень у числі випадків мають істотні відмінності, пристрій формування матриць Адамара переважно реалізуються програмними засобами. В статті проаналізовані ансамблеві характеристики ортогональних систем сигналів для запропонованих алгоритмів побудови. Показано, щзо кількість алгоритмів побудови ортогональних систем сигналів суттєво зростає.

Ключові слова: ортогональні системи сигналів, матрищі Адамара, ансамблеві характеристики, системи зв'язку та управління, алгоритми побудови сигналів, дискретні сигнали.

\section{Вступ}

Постановка проблеми. Рішення проблеми забезпечення необхідної якості передачі інформації в системах зв'язку і управління пов'язують з сигналами, що володіють необхідним кореляційними, ансамблевими та структурними властивостями. Серед відомих систем сигналів, які вже знайшли застосування, значний інтерес представляють ортогональні дискретні сигнали (ОДС). Інтерес до ортогональних сигналів обумовлений тим, що їх використання дозволяє зменшити ймовірність помилки в системі зв'язку і управління, підвищує їх пропускну здатність [1].

В середовищі ортогональних сигналів особливої уваги заслуговують ОДС, що побудовані на основі матриць Адамара. Однак на сьогоднішній день розроблені алгоритми побудови і дослідження властивостей тільки ОДС, що побудовані на основі матриць Адамара 3 числом елементів $L=2^{r}$, де $r=2,3,4 \ldots$ [2]. В той же час ортогональні сигнали можуть бути побудовані для будь-яких значень $L \equiv 0(\bmod 4)$.

Аналіз останніх досліджень і публікацій. Алгоритми побудови ортогональних сигналів на основі матриць Адамара для $L=2^{r}$ широко розглянуті в літературі [3-4].

В [5] показано, що ортогональні сигнали можуть бути побудовані для $L \equiv 0(\bmod 4)$, але правил побудови не приводиться. В [6] проводиться аналіз ансамблевих характеристик ОДС, але також для $L=2^{r}$. В [7] запропонований алгоритм збільшення ансамблевих характеристик ОДС на основі похідних систем сигналів.

Мета статті - розробка алгоритмів синтезу ОДС $3 L \equiv 0(\bmod 4)$ і дослідження ансамблевих характеристик таких систем для захисту від навмисних структурних перешкод в системах зв'язку та управління.

\section{Виклад основного матеріалу}

Нехай $\{W\}$ є ортогональною системою дискретних сигналів. Позначимо $j$-й словник ортогональних дискретних сигналів $\left\{W_{j}\right\}_{o p m}$ як,$j=\overline{1, M}$, де $M$ - число різних словників . Тоді $i$ та $(i, v=\overline{1, L})$

ОДС $W^{j}$ словника являються ортогональними, якщо виконується умова:

$$
\sum_{k=1}^{L}\left\{W_{i}^{j}\right\}_{k}\left\{W_{v}^{j}\right\}_{k}=\left\{\begin{array}{l}
0 \text { nри } i \neq v \\
L \text { при } i=v
\end{array}\right.
$$

Ортогональні дискретні сигнали аналітично можуть бути задані різними способами. Найбільш зручною є форма подання ОДС $з$ використанням функцій Адамара, під якими розуміють послідовно- 
сті з $L$ прямокутних імпульсів з амплітудою, що дорівнює одиниці, і полярністю, яка визначається елементами рядків матриці Адамара порядку $m$, i т. д.

$$
A_{m}=\left|\begin{array}{cccc}
a_{11} & a_{12} & \ldots & a_{1 m} \\
a_{21} & a_{22} & \ldots & a_{2 m} \\
\ldots & \ldots & \ldots & \ldots \\
a_{m 1} & a_{m 2} & \ldots & a_{m n}
\end{array}\right| .
$$

Наведені нижче твердження дають умови існування ОДС для різних значень тривалості $L$.

Твердження 1. Нехай $\left\{W_{m}^{j}\right\} \in$ матриця Адамара, причому $m>3$, тоді вона існує (матриця Адамара порядку $m)$, якщо $m=L=0(\bmod 4)$.

Доведення твердження 1 .

Нехай $A_{m} \quad$ - матриця Адамара $(m>3)$, тоді справедливе наступне співвідношення [3]:

$$
\begin{gathered}
\sum_{j=0}^{m-1}\left(a_{1 j}+a_{2 j}\right)\left(a_{1 j}+a_{3 j}\right)= \\
=\sum_{j=0}^{m-1}\left(a_{1 j} a_{1 j}+a_{2 j} a_{1 j}+a_{1 j} a_{3 j}+a_{2 j} a_{3 j}\right)= \\
=\sum_{j=0}^{m-1} a_{1 j}^{2}+\sum_{j=0}^{m-1} a_{2 j} a_{1 j}+\sum_{j=0}^{m-1} a_{1 j} a_{3 j}+\sum_{j=0}^{m-1} a_{2 j} a_{3 j}= \\
=\sum_{j=0}^{m-1} a_{1 j}^{2}=m . \\
A_{m_{2}}=A_{2}=\left|\begin{array}{rr}
1 & 1 \\
1 & -1
\end{array}\right| .
\end{gathered}
$$

Так як $\left(a_{1}+a_{2}\right)\left(a_{1}+a_{3}\right)$ дорівнює або 4 , або 0 , знаходимо: $m=4 t, t=1,2 \ldots$ Звідси випливає, що $m$ ділиться на 4.

Твердження 2. Існують матриці Адамара першого і другого порядку:

$$
\left\{W_{1}^{j}\right\}=|1|,\left\{W_{2}^{j}\right\}=\left|\begin{array}{rr}
1 & 1 \\
1 & -1
\end{array}\right| .
$$

Доведення твердження 2.

Позначимо елементи першого рядка як сигнал $W_{1}$, а елементи другого рядка як сигнал $W_{2}$. Використовуючи вираз (1), зрозуміло, що результат суми елементів $W_{1}$ i $W_{2}$ дорівнює нулю.

Твердження 3. Якщо $M=2^{r}$, то існує матриця Адамара порядку $2^{r}$.

Доведення твердження 3.

Нехай $r=1$, тоді $m=2^{1}=2$. У цьому випадку існує матриця Адамара, обумовлена твердженням 2. При $r<1 m \equiv 0(\bmod 4)$, так як число $2^{r}$ завжди містить співмножник, рівний 4.

Твердження 4. Якщо існують матриці Адамара
$\left\{W_{m_{1}}\right\}$ i $\left\{W_{m_{2}}\right\}$ відповідно $m_{1}$ і $m_{2}$ порядків, то існує матриця Адамара $\left\{W_{m}\right\}$, де $m=m_{1} m_{2}$, отримана з матриці $\left\{W_{m_{1}}\right\}$ підстановкою $\left\{W_{m_{2}}\right\}$ замість $(+1)$ i $\left\{-W_{m_{2}}\right\}$ замість $(-1)$.

Доведення твердження 4.

Нехай матриця Адамара $A_{m_{1}}$ порядку $m_{1}$ складається $з$ елементів $a_{h_{1}}\left(0 \leq h, 1 \leq m_{1}-1\right)$, а матриця Адамара $A_{m_{2}}$, порядку $m_{2}$ складається 3 елементів $b_{i j}\left(0 \leq i, j \leq m_{2}-1\right)$.

Створимо матрицю з елементів:

$$
\begin{gathered}
c_{s t}\left(s=h m_{2}+i, t=l m+j, 0 \leq h, 1 \leq m_{1}-1 ;\right. \\
\left.0 \leq i, j \leq m_{2}-1,0 \leq s, t \leq m_{1} m_{2}-1\right),
\end{gathered}
$$

таким чином, щоб $m_{2} c_{s t}=a_{h_{1}} b_{i j}$.

Візьмемо $s_{1}=h_{1} m_{2}+i_{2} s_{2}=h_{1} m_{2}+i_{2}, \quad$ причому $s_{1} \neq s_{2}$, тоді:

$$
\begin{gathered}
\sum_{t=0}^{m_{1} m_{2}} c_{s_{1}} t c_{s_{2}} t=\sum_{j=0}^{m_{2}-1} \sum_{i=0}^{m_{1}-1} a_{h_{1}} l b_{i_{1}} j a_{h_{2}} l b_{i_{2}} j= \\
=\sum_{j=0}^{m_{2}-1} b_{i_{1}} j b_{i_{2}} j \sum_{i=0}^{m_{1}-1} a_{h_{1}} l a_{h_{2}} l=0 .
\end{gathered}
$$

Приклад:

нехай

$$
A_{m_{2}}=A_{4}=\left|\begin{array}{rrrr}
1 & 1 & 1 & 1 \\
1 & -1 & 1 & -1 \\
1 & 1 & -1 & -1 \\
1 & -1 & -1 & 1
\end{array}\right| .
$$

Тоді підставляючи в матрицю $A_{m_{1}}$ замість 1 елементи матриці $A_{m_{2}}$ і замість $(-1)$ елементи матриці $-A_{m_{2}}$, отримаємо:

$$
\begin{gathered}
A_{m}=A_{8}=\left|\begin{array}{lllllll}
A_{m_{2}} & A_{m_{2}} \\
A_{m_{2}} & -A_{m_{2}}
\end{array}\right| ; \\
A_{8}=\left|\begin{array}{rrrrrrrr}
-1 & -1 & -1 & -1 & -1 & -1 & -1 & -1 \\
-1 & -1 & -1 & -1 & -1 & -1 & -1 & -1 \\
-1 & -1 & -1 & -1 & -1 & -1 & -1 & -1 \\
-1 & -1 & -1 & -1 & -1 & -1 & -1 & -1 \\
-1 & -1 & -1 & -1 & -1 & -1 & -1 & -1 \\
-1 & -1 & -1 & -1 & -1 & -1 & -1 & -1 \\
-1 & -1 & -1 & -1 & -1 & -1 & -1 & -1 \\
-1 & -1 & -1 & -1 & -1 & -1 & -1 & -1
\end{array}\right| .
\end{gathered}
$$

Твердження 5. Для будь-якого простого числа $p \equiv 3(\bmod 4)$ існує матриця Адамара порядку $m=p+1$. 
Доведення твердження 5.

Для побудови матриці використовується символ Лежандра [6]. Він визначається для всіх $n$, що не діляться на $p$, і задається таким чином: $(n / p)=1$, якщо $n$ - квадратичне віднімання по модулю $p$, тобто, якщо $n \equiv x^{2}(\bmod p) ; \quad(n / p)=-1, \quad$ якщо $n \quad x^{2}(\bmod p) . \quad \not \equiv$

Правило побудови матриці Адамара: $a_{i j}=1$, якщо $i=0$ або $j=0 ; a_{i j}=i-j / p$, якщо $l \leq i \leq p, l \leq j \leq p, i \neq j ; a_{i j}=-1$, якщо $l \leq i \leq p ;$

Тоді, якщо $i_{1}$ та $i_{2}$ різні і більші 0, маємо:

$$
\begin{gathered}
\sum_{j=0}^{p} a_{i_{1} j} a_{i_{2} j}=a_{i_{1} i_{1}} a_{i_{2} i_{2}}+a_{i_{1} i_{2}} a_{i_{2} i_{2}}+a_{i_{1} 0} a_{i_{2} 0+} \\
+\sum_{j=1}^{p}\left(\frac{j-i_{1}}{p}\right)\left(\frac{j-i_{2}}{p}\right)=\left(\frac{i_{1}-i_{2}}{p}\right)-\left(\frac{i_{2}-i_{1}}{p}\right)+ \\
+1 \sum_{j=1}^{p}\left(\frac{i-i_{1}}{p}\right)\left(\frac{i-i_{2}}{p}\right) \\
j=i_{1}, j \neq i_{2} .
\end{gathered}
$$

3 огляду на властивості символу Лежандра [3]:

$$
\sum_{j=0}^{p-1}\left(\frac{j-i}{p}\right)=0 ; \frac{i_{2}-i_{1}}{p}=-\frac{i_{1}-i_{2}}{p}
$$

отримаємо:

$$
\sum_{j=0}^{p} a_{i_{1}} j a_{i_{2} j}=0 .
$$

Нарешті, якщо $i_{1}=0$ то:

$$
\begin{gathered}
\sum_{j=0}^{p} a_{0 j} a_{i j}=\sum_{j=0}^{p} a_{i j}=a_{i j}+a_{i 0} \sum_{i=1}^{p}\left(\frac{j-i}{p}\right)= \\
=-1+1+0=0 .
\end{gathered}
$$

Таким чином, рядки матриці $A_{m}$ ортогональні, а отже, вона є матрицею Адамара. Приклад.

Нехай $m=7+1$, тоді:

$$
A_{m}=\left|\begin{array}{rrrrrrrr}
-1 & -1 & -1 & -1 & -1 & -1 & -1 & -1 \\
-1 & -1 & -1 & -1 & -1 & -1 & -1 & -1 \\
-1 & -1 & -1 & -1 & -1 & -1 & -1 & -1 \\
-1 & -1 & -1 & -1 & -1 & -1 & -1 & -1 \\
-1 & -1 & -1 & -1 & -1 & -1 & -1 & -1 \\
-1 & -1 & -1 & -1 & -1 & -1 & -1 & -1 \\
-1 & -1 & -1 & -1 & -1 & -1 & -1 & -1 \\
-1 & -1 & -1 & -1 & -1 & -1 & -1 & -1
\end{array}\right| .
$$

Твердження 6. Якщо $m=2^{k}(p+1)$, де $k$-ціле, а $p$ - просте число, то існує матриця Адамара порядку $2^{k}(p+1)$.
Доведення твердження 6 пропонується читачеві провести самостійно, використовуючи матеріали попередніх тверджень.

Твердження 7 [3]. Якщо $m=2^{k} p(p+1)$, де $p \equiv 3(\bmod 4)-$ просте число, то існує матриця Адамара порядку $2^{k} p(p+1)$.

Твердження 8. Якщо $m=2^{k}\left(p^{n}+1\right)$, де $p-$ непарне просте число, то існує матриця Адамара $2^{k}\left(p^{n}+1\right)$.

Твердження 9. Якщо $m=m^{*}\left(m^{*}-1\right)$, де $m^{*}=2^{r}\left(p_{1}+1\right)\left(p_{2}+1\right) \ldots\left(p_{i}+1\right)$, причому $p_{i}+1 \equiv 0(\bmod 4)$, то існує матриця Адамара порядку $m^{*}\left(m^{*}-1\right)$.

Твердження 10. Якщо існує матриця Адамара порядку $m_{1}$ i $m_{2} \geq 2$, то існує матриця Адамара порядку $m=m_{1} m_{2} p(p+1)$, де $p$-просте непарне число.

Твердження 11. Якщо існують матриці Адамаpa $m_{1}$ i $m_{2}$, a $p_{2}=p_{1}+4$, причому $p_{1}$ і $p_{2}$ прості, то існує матриця порядку $m_{1} m_{2} p_{1}\left(p_{2}-1\right)$.

Твердження 12. Якщо $p_{2}=p_{1}+2, p_{1}$ i $p_{2}-$ прості, то існує матриця Адамара порядку $\left(p_{1}+1\right)^{2}=\left(p_{2}-1\right)^{2}$.

Твердження 13. Якщо $m=13 k$, причому $k=4 t, t=1,2, \ldots, \quad$ то існує матриця Адамара порядку $m$.

Твердження 14. Якщо $m=2^{r}$, то існує матриця Адамара, рядками якої являються автоморфізми ЛРПМ, в якості $2^{r}$ елемента яких використовується символ $1(-1)$.

Докази тверджень 6-14 аналогічні доказам тверджень 1-5.

Твердження 15. Якщо $M<200$, то існує метод Вільямса, з використанням якого можуть бути побудовані матриці Адамара порядку 92, 116, 184.

Твердження 16 [5]. Існує матриця порядку $m=172$.

Проаналізуємо ансамблеві характеристики ОДС системи Адамара. 3 цією метою визначимо число різних матриць Адамара, які можна побудувати для всіx $L \equiv 0(\bmod 4)$.

3 тверджень 3 і 14 випливає, що матриці Адамара порядку $2^{r}$ можуть бути побудовані або з використанням ізоморфізмів ЛРПМ, або з використанням матриці другого порядку 


$$
\left\{W_{2}\right\}=\left|\begin{array}{rr}
1 & 1 \\
1 & -1
\end{array}\right|,
$$

при цьому

$$
\begin{aligned}
\left\{W_{3}\right\} & =\left\|\begin{array}{lr}
W_{2} & W_{2} \\
W_{2} & -W_{2}
\end{array}\right\|, \ldots\left\{W_{m}\right\}= \\
& =\left\|\begin{array}{ll}
W_{m-1} & W_{m-1} \\
W_{m-1} & -W_{m-1}
\end{array}\right\| .
\end{aligned}
$$

Матриця виду (4) 3 точністю до перестановки рядків може бути сформована єдиним чином [3]. Число не інверсних ізоморфізмів $M_{m}^{\prime}$ ЛРПМ визначається функцією Ейлера [3]

$$
M_{m}^{\prime}=\frac{\varphi\left(2^{m}-1\right)}{2 m} .
$$

Однак матриця $m$ порядку може бути побудована з використанням ізоморфізмів ЛРПМ з числом елементів $2^{m-1}-1$ (твердження 14) і матриці виду (4).

У цьому випадку загальна кількість різних матриць Адамара буде визначатися виразом

$$
M_{m}=M_{m}^{\prime}+M_{m-1}^{\prime}+M_{m-2}^{\prime}+\ldots+M_{2}^{\prime}+1 .
$$

Число ізоморфних матриць Адамара, побудованих відповідно до твердження 5, дорівнює 1. Дійсно, як показано в [6], якщо $\rho \equiv 3(\bmod 4)$, а $m=\rho+1$, то множина коефіцієнтів $(k-e) / \rho-\epsilon д и-$ на.

Ансамбль ізоморфних матриць Адамара, побудованих відповідно до тверджень 4, 6 і 11 зважаючи на побудову їх методом простої підстановки, визначається відтворенням ансамблів матриць, використовуваних в якості базових:

$$
M=M_{m_{1}} M_{m_{2}} .
$$

Аналогічно для випадку, коли матриця Адамара будується на основі більш ніж двох матриць, розмірність ансамблю ізоморфних матриць визначається із співвідношення:

$$
M=\prod_{i=1}^{k} M_{m_{1}} .
$$

Вивчення ансамблевих характеристик матриць Адамара зважаючи на специфіку методів їх побудови вимагає важко реалізованих обчислювальних витрат. Як випливає з [5], матриці Адамара розглянутих порядків можуть бути побудовані одним або декількома способами.

У табл. 1 наведені значення ансамблевих характеристик ізоморфізмів ОДС, побудованих з використанням матриць Адамара для деяких значень $L$.

Таблиця 1

Ансамблеві характеристики ОДС

\begin{tabular}{|c|c|c|c|c|c|}
\hline$L$ & 12 & 24 & $\ldots$ & 9000 & 10000 \\
\hline$M$ & 3 & 4 & $\ldots$ & 12 & 10 \\
\hline
\end{tabular}

Джерело: [8, С. 460].

Аналіз даних табл. 1 показує, що при використанні ОДС, побудованих на основі сформульованих тверджень, досягається збільшення значень ансамблевих характеристик.

\section{Висновки}

Таким чином для абсолютної більшості значень $L \equiv 0(\bmod 4)$ існують матриці Адамара.

Алгоритми побудови матриць Адамара для різних значень $L$ в ряді випадків мають суттєві відмінності.

Запропоновані алгоритми побудови ортогональних систем сигналів мають кращі ансамблеві характеристики та можуть бути застосовані в системах зв'язку та управління, де задачі захисту від навмисних структурних перешкод вирішуються на фізичному рівні.

\section{Список літератури}

1. Paterson K. Sequences For OFDM and Multi-code CDMA: two problems in algebraic coding theory. Sequences and their applications : Second Int. Conference, Bergen, Norway, 13-17 May 2018. P. 46-71. URL: https://webcache.googleusercontent.com/search?q=cache:PkaPfoQFrX8J:https://www.isg.rhul.ac.uk/ kp/twoprobs.ps+\&cd=2\&h $\mathrm{l}=\mathrm{uk} \& \mathrm{ct}=\mathrm{clnk} \& \mathrm{gl}=\mathrm{ua}$.

2. Hall M. Jr. Hadamard matrices of order 16. Research Summary. 2017. Vol. 1. No. 36(10). P. 21-26.

3. Rao K. Discrete Cosine Transform: Algorithms, Advantages, Applications. New York : Academic. Press, 2019. 512 p.

4. Hayes B. Third base. Computing science. 2016. Vol. 89. No. 6. P. 490-494.

5. Приходько С. І., Кузнєцов А. А., Гусєв С. А., Кужель І. Є. Алгебраїчна побудова несистематических згортальних кодів. Системи обробки інформаиї. 2004. № 8(36). С. 170-175.

6. Franks L. Signal Theory. Hoboken : Prentice Hall, 2017. 318 p.

7. Hawkes P. Advances in Electronics and Electron Physics. Academic Press. 2020. Vol. 88. P. 365.

8. Sokolov A. Regular synthesis method of the sequences of length $\mathrm{N}=24$ with optimal PAPR of Walsh-Hadamard spectrum. Far East Journal of Electronics and Communications. 2018. Vol. 16. No. 2. P. 459-469. http://dx.doi.org/10.17654/EC016020459.

9. Sloane N. A Library of Hadamard Matrices: web site. URL: http://neilsloane.com/hadamard/ (accessed 26.08.2021).

10. Horadam K. Hadamard matrices and their applications. Princeton : Princeton University Press, 2016. 278 p.

11. Превисокова Н., Метод обробки інформації на основі дискретного ортогонального перетворення. Вісник Хмельницького національного університету. 2018. № 2(146). С. 149-156. 
12. Приходько С. І., Гусєв С. А., Зубенко В. А. Розробка каскадних кодових конструкцій з покращеними властивостями. Системи обробки інформації. 2011. № 2(92). С. 119-128.

\section{Відомості про авторів:}

Стасєв Юрій Володимирович

доктор технічних наук професор

професор кафедри Харківського національного

університету Повітряних Сил ім. І. Кожедуба,

Харків, Україна

https://orcid.org/0000-0002-3893-0313

\section{Сійчук Аліна Юріївна}

курсант Харківського національного університету

Повітряних Сил ім. І. Кожедуба,

Харків, Україна

https://orcid.org/0000-0003-3654-6972

\section{Костиленко Кирило Юрійович}

курсант Харківського національного університету Повітряних Сил ім. І. Кожедуба,

Харків, Україна

https://orcid.org/0000-0002-8679-5777

\section{Манакова Ольга Володимирівна}

курсант Харківського національного університету

Повітряних Сил ім. І. Кожедуба,

Харків, Україна

https://orcid.org/0000-0001-9209-1840

\section{Information about the authors:}

Yuriy Stasiev

Doctor of Technical Sciences Professor

Professor of Ivan Kozhedub Kharkiv

National Air Force University,

Kharkiv, Ukraine

https://orcid.org/0000-0002-3893-0313

\author{
Alina Siichuk \\ Cadet of Ivan Kozhedub Kharkiv \\ National Air Force University, \\ Kharkiv, Ukraine \\ https://orcid.org/0000-0003-3654-6972
}

\author{
Kirill Kostylenko \\ Cadet of Ivan Kozhedub Kharkiv \\ National Air Force University, \\ Kharkiv, Ukraine \\ https://orcid.org/0000-0002-8679-5777
}

\section{Olha Manakova}

Cadet of Ivan Kozhedub Kharkiv

National Air Force University,

Kharkiv, Ukraine

https://orcid.org/0000-0001-9209-1840

\section{АЛГОРИТМЫ ПОСТРОЕНИЯ ДИСКРЕТНЫХ ОРТОГОНАЛЬНЫХ СИГНАЛОВ НА ОСНОВЕ МАТРИЦ АДАМАРА}

Ю.В. Стасев, А.Ю. Сийчук, К.Ю. Костыленко, О.В. Манакова

В статье предложены алгоритмы построения ортогональных систем сигналов на основе матрии Адамара с числом элементов $L \equiv 0(\bmod 4)$. Реализация этих алгоритмов позволяет более гибко использовать дискретные сигналь в случаях изменения энергетических, временных и частотных ресурсов систем связи и управления. В статье сформулированы и доведены утверждения, что является математической основой построения ортогональных сигналов с числом элементов $L \equiv 0(\bmod 4)$. Показано, что практически для всех значений $L \equiv 0(\bmod 4)$ существует алгоритм построения ортогональных сигналов. Алгоритмы построения матриц Адамара для различных значений в ряде случаев имеют существеннье различия. Анализ способов и алгоритмов построения ортогональных систем сигналов позволяет сделать следующие выводы, что для абсолютного больиинства значений существуют матриць Адамара, алгоритмы построения матрии Адамара для различных значений в ряде случаев имеют существенные различия, устройство формирования матрии Адамара преимущественно реализуется программными средствами. Изучение ансамблевых характеристик матриц Адамара, учитывая специфику методов их построения, требует сложно реализуемьх вычислительных затрат. Матриць Адамара рассмотренных порядков могут быть построены одним или несколькими способами. Результаты исследований показывают, что при использовании ортогональных дискретных сигналов, построенных на основе сформулированных утверждений, достигается увеличение значений ансамблевых характеристик. Предложенные алгоритмы построения ортогональных систем сигналов имеют лучиие ансамблевые характеристики и могут быть применены в системах связи и управления, где задачи защчиты от преднамеренных структурных препятствий решаются на физическом уровне. Разработаны рекомендации по комплектованию различных алгоритмов построения ортогональных систем сигналов для получения сигналов с нужным числом элементов. В статье проанализированы ансамблевые характеристики ортогональных систем сигналов для предложенных алгоритмов построения. Показано, что количество алгоритмов построения ортогональных систем сигналов существенно возрастает.

Ключевые слова: ортогональные системы сигналов, матрицы Адамара, ансамблевые характеристики, системы связи и управления, алгоритмы построения сигналов, дискретные сигналь. 


\title{
ALGORITHMS FOR CONSTRUCTING DISCRETE ORTHOGONAL SIGNALS BASED ON HADAMARD MATRICES
}

\author{
Yu. Stasiev, A. Siichuk, K. Kostylenko, O. Manakova
}

The article proposes algorithms for constructing orthogonal signal systems based on Hadamard matrices with the number of elements $L \equiv 0(\bmod 4)$. The implementation of these algorithms allows more flexible use of discrete signals in cases of changes in the energy, time and frequency resources of communication and control systems. The article formulates and brings the statement that is the mathematical basis for constructing orthogonal signals with the number of elements $L \equiv 0(\bmod 4)$. It is shown that for almost all values of $L \equiv 0(\bmod 4)$ there is an algorithm for constructing orthogonal signals. Algorithms for constructing Hadamard matrices for different values in a number of cases have significant differences. An analysis of the methods and algorithms for constructing the orthogonal discrete signals allows us to draw the following conclusions that for the absolute majority of values there are Hadamard matrices, the algorithms for constructing Hadamard matrices for various values in some cases have significant differences, the device for forming Hadamard matrices is mainly implemented by software. The study of the ensemble characteristics of Hadamard matrices, taking into account the specifics of the methods for their construction, requires computational costs that are difficult to implement. Hadamard matrices of the considered orders can be constructed in one or several ways. The research results show that when using orthogonal discrete signals built on the basis of the formulated statements, an increase in the values of ensemble characteristics is achieved. The proposed algorithms for constructing orthogonal signal systems have better ensemble characteristics and can be applied in communication and control systems, where the problems of protection against deliberate structural obstacles are solved at the physical level. Recommendations have been developed for the acquisition of various algorithms for constructing orthogonal signal systems to obtain signals with the required number of elements. The article analyzes the ensemble characteristics of orthogonal signal systems for the proposed construction algorithms. It is shown that the number of algorithms for constructing orthogonal signal systems increases significantly.

Keywords: orthogonal signal systems, Hadamard matrices, ensemble characteristics, communication and control systems, signal construction algorithms, discrete signals. 